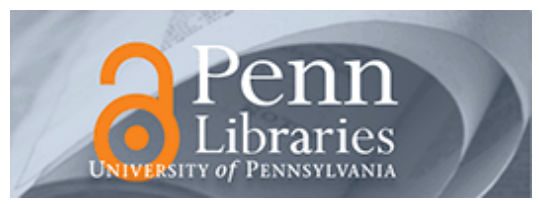

University of Pennsylvania ScholarlyCommons

December 2005

\title{
Theory of simultaneous control of orientation and translational motion of nanorods using positive dielectrophoretic forces
}

\author{
Brian Edwards \\ University of Pennsylvania \\ Nader Engheta \\ University of Pennsylvania, engheta@ee.upenn.edu \\ Stephane Evoy \\ University of Alberta
}

Follow this and additional works at: https://repository.upenn.edu/ese_papers

\section{Recommended Citation}

Brian Edwards, Nader Engheta, and Stephane Evoy, "Theory of simultaneous control of orientation and translational motion of nanorods using positive dielectrophoretic forces", . December 2005.

Copyright 2005 American Institute of Physics. This article may be downloaded for personal use only. Any other use requires prior permission of the author and the American Institute of Physics. Reprinted in Journal of Applied Physics, Volume 98, Issue 12, Article 124314, December 30, 2005, 7 pages.

Publisher URL: http://dx.doi.org/10.1063/1.2148627

This paper is posted at ScholarlyCommons. https://repository.upenn.edu/ese_papers/265

For more information, please contact repository@pobox.upenn.edu. 


\title{
Theory of simultaneous control of orientation and translational motion of nanorods using positive dielectrophoretic forces
}

\author{
Abstract \\ The manipulation of individual submicron-sized objects has been the focus of significant efforts over the \\ last few years. A method to arbitrarily move and orient a set of rod-shaped conductive particles in a \\ region defined by a set of electrodes using positive dielectrophoretic forces is presented. While the \\ orientation of each particle is directly specified through the angle of the local electric field, its position is \\ indirectly controlled through the applied force. Each electrode is approximated as an unknown point \\ charge and an induced dipole. Since each induced dipole results from the combination of all other \\ sources, a set of linear constraints are derived to enforce the self-consistency of the system. Additionally, \\ the force and orientation of each particle also form an additional set of linear constraints. This combined \\ set of constraints is then solved numerically to yield the sources required to induce the desired \\ orientation and motion of each particle. It is observed that the minimum number of electrodes that can be \\ used to control a set of $\mathrm{N}$ particles is $4 N+1$. Numerical simulations demonstrate that the control of a \\ single nanorod (diameter of $70 \mathrm{~nm}$; length of $1.4 \mu \mathrm{m}$ ) in the midst of a realistic electrode array can be \\ accomplished under practical conditions. In addition, such control of orientation and motion can be \\ achieved over an ample region in the vicinity of each rod.

\section{Comments} \\ Copyright 2005 American Institute of Physics. This article may be downloaded for personal use only. Any \\ other use requires prior permission of the author and the American Institute of Physics. Reprinted in \\ Journal of Applied Physics, Volume 98, Issue 12, Article 124314, December 30, 2005, 7 pages. \\ Publisher URL: http://dx.doi.org/10.1063/1.2148627
}




\title{
Theory of simultaneous control of orientation and translational motion of nanorods using positive dielectrophoretic forces
}

\author{
Brian Edwards and Nader Engheta \\ Department of Electrical and Systems Engineering, University of Pennsylvania, Philadelphia, \\ Pennsylvania 19104 \\ Stephane Evoy ${ }^{\text {a) }}$ \\ Department of Electrical and Computer Engineering and National Institute for Nanotechnology, \\ University of Alberta, Edmonton AB T6G 2V4, Canada
}

(Received 1 June 2005; accepted 9 November 2005; published online 30 December 2005)

\begin{abstract}
The manipulation of individual submicron-sized objects has been the focus of significant efforts over the last few years. A method to arbitrarily move and orient a set of rod-shaped conductive particles in a region defined by a set of electrodes using positive dielectrophoretic forces is presented. While the orientation of each particle is directly specified through the angle of the local electric field, its position is indirectly controlled through the applied force. Each electrode is approximated as an unknown point charge and an induced dipole. Since each induced dipole results from the combination of all other sources, a set of linear constraints are derived to enforce the self-consistency of the system. Additionally, the force and orientation of each particle also form an additional set of linear constraints. This combined set of constraints is then solved numerically to yield the sources required to induce the desired orientation and motion of each particle. It is observed that the minimum number of electrodes that can be used to control a set of $N$ particles is $4 N+1$. Numerical simulations demonstrate that the control of a single nanorod (diameter of $70 \mathrm{~nm}$; length of $1.4 \mu \mathrm{m}$ ) in the midst of a realistic electrode array can be accomplished under practical conditions. In addition, such control of orientation and motion can be achieved over an ample region in the vicinity of each rod. (C) 2005 American Institute of Physics. [DOI: 10.1063/1.2148627]
\end{abstract}

\section{INTRODUCTION}

The ability to manipulate individual nanostructures is necessary for the characterization of their electrical and mechanical behaviors, analysis of their response to outside agents and stimuli, and, more importantly, their eventual integration into functional systems. The ability to arbitrarily move single entities would also prove useful for the heterogeneous integration of nanoelectronic devices, ${ }^{1}$ studies of nanofluidics, ${ }^{2}$ and laboratory on a chip technologies. ${ }^{3}$ While optical tweezers ${ }^{4,5}$ allows for such manipulation, it is limited in resolution due to physical constraints on laser spot size. Additionally atomic force microscopy can be used to position nanoscale particles but involves a complex apparatus. ${ }^{6}$ Magnetic tweezers also allows arbitrary positioning of particles, but this approach is constrained to magnetic entities.

A large amount of work has been done lately in the field of electrokinetics, ${ }^{8-10}$ specifically with respect to the manipulation of individual particles. ${ }^{3}$ For instance, since the pioneering work of Pohl, ${ }^{11}$ there has been substantial interest towards the development of dielectrophoretic-based approaches to the manipulation of micron- and nanoscale objects. These include negative dielectrophoretic trapping, ${ }^{12-14}$ electrorotation, ${ }^{15}$ positive dielectrophoresis, ${ }^{16-18}$ and traveling-wave dielectrophoresis. ${ }^{19}$ While relatively easy to implement, these techniques are usually limited to the positioning of particles within electrode gaps, and thus limited to accuracies defined by the electrode spacings themselves.

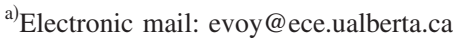

While particles have been manipulated within the space between electrodes, to our knowledge it has never been done arbitrarily. ${ }^{20}$

We propose an alternate approach to the usage of dielectrophoretic forces for manipulation of nanoparticles. This approach enables the arbitrary manipulation of multiple rodshaped conducting particles within a region defined by an electrode array. We specifically examine the electrostatics of a set of coplanar particles and electrodes required for such control. While the orientations of the individual particles are readily controlled through the angle of the electric field, their positions can only be indirectly manipulated through an applied force. We thus derive the conditions that the field must satisfy at each particle's position to produce the desired individual motion and orientation. We demonstrate that a desired orientation and force can be imposed on $N$ particles providing that there are $4 N+1$ electrodes, and that this manipulation can be accomplished under realistic conditions within a region of reasonable dimension.

\section{DEVELOPMENT OF METHOD}

Suppose one wishes to control the position and orientation of a set of polarizable rod-shaped particles using an applied electric field generated by a set of electrodes in the same plane as the particles (Fig. 1). Each rod can be modeled as an induced dipole at location $\mathbf{R}_{j}$, where $j$ goes from 1 to $N$, and possesses a polarizability $\alpha_{\text {rod }}$ that is negligible in all directions except along its axis. A potential-energy minimum exists with respect to orientation when the rod is aligned 
with the local electric field. Assuming that the field changes slowly enough so that all inertial and drag terms are negligible, controlling the orientation of the rod is therefore readily accomplished by controlling the direction of the electric field $\mathbf{E}$ at that location. In the following discussion, the rod is indeed assumed to be oriented along the field, allowing the dipole moment to be introduced as $\mathbf{p}_{\text {rod }}=\alpha_{\text {rod }} \mathbf{E}$.

It would be convenient if there was an analogous method for controlling the position of the particle, i.e., through a potential-energy minimum with respect to the spatial coordinates. However, such direct control is physically impossible for the reasons outlined below.

The potential energy $U$ of a single dipole is given by

$$
\begin{aligned}
U & =-\mathbf{p}_{\mathrm{rod}} \cdot \mathbf{E} \\
& =-\left.\alpha_{\mathrm{rod}}(\mathbf{E} \cdot \mathbf{E})\right|_{\mathbf{R}_{j}} \\
& =-\left.\alpha_{\mathrm{rod}}\left[\left(\frac{\partial V}{\partial x}\right)^{2}+\left(\frac{\partial V}{\partial y}\right)^{2}+\left(\frac{\partial V}{\partial z}\right)^{2}\right]\right|_{\mathbf{R}_{j}},
\end{aligned}
$$

where $V$ is the voltage field. The existence of an energy minimum would require that the first derivatives of the potential energy be equal to zero with respect to all directions, and that the second derivatives be positive with respect to all directions. It is sufficient to show that

$$
\operatorname{Max}\left[\frac{\partial^{2} U}{\partial x^{2}}+\frac{\partial^{2} U}{\partial y^{2}}+\frac{\partial^{2} U}{\partial z^{2}}\right] \leqslant 0
$$

to prove that it is impossible to simultaneously satisfy $\partial^{2} U / \partial x^{2}>0, \partial^{2} U / \partial y^{2}>0$, and $\partial^{2} U / \partial z^{2}>0$, and thus impossible to create a potential-energy minimum with respect to space. If the field is static and in a source-free region, it must also satisfy Laplace's equation,

$$
\frac{\partial^{2} V}{\partial x^{2}}+\frac{\partial^{2} V}{\partial y^{2}}+\frac{\partial^{2} V}{\partial z^{2}}=0
$$

By taking the first derivatives of Laplace's equation to get three linear constraints, Eq. (2) is constrained by

$$
\begin{aligned}
& \left.\frac{\partial^{3} V}{\partial x^{3}}\right|_{\mathbf{R}_{j}}+\left.\frac{\partial^{3} V}{\partial x \partial y^{2}}\right|_{\mathbf{R}_{j}}+\left.\frac{\partial^{3} V}{\partial x \partial z^{2}}\right|_{\mathbf{R}_{j}}=0, \\
& \left.\frac{\partial^{3} V}{\partial y \partial x^{2}}\right|_{\mathbf{R}_{j}}+\left.\frac{\partial^{3} V}{\partial y^{3}}\right|_{\mathbf{R}_{j}}+\left.\frac{\partial^{3} V}{\partial y \partial z^{2}}\right|_{\mathbf{R}_{j}}=0, \\
& \left.\frac{\partial^{3} V}{\partial z \partial x^{2}}\right|_{\mathbf{R}_{j}}+\left.\frac{\partial^{3} V}{\partial z \partial y^{3}}\right|_{\mathbf{R}_{j}}+\left.\frac{\partial^{3} V}{\partial z^{3}}\right|_{\mathbf{R}_{j}}=0 .
\end{aligned}
$$

By expressing potential energy in Eq. (2) in terms of the voltage field through Eq. (1), treating each of the local derivatives to be the variables, and maximizing the goal function in Eq. (2) by changing these variables under the constraints in Eq. (4), it can be shown that

$$
\operatorname{Max}\left[\frac{\partial^{2} U}{\partial x^{2}}+\frac{\partial^{2} U}{\partial y^{2}}+\frac{\partial^{2} U}{\partial z^{2}}\right]=0,
$$

and, therefore, it seems impossible to contain an induced dipole in a source-free region with a static field.

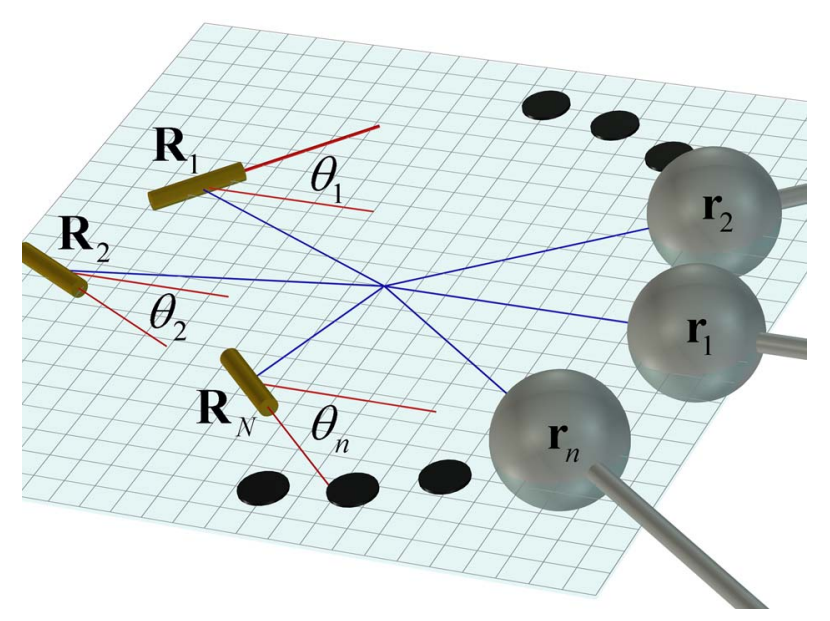

FIG. 1. (Color online) An array of $n$ electrodes in the same plane as $N$ arbitrarily positioned and oriented particles. The black dots represent continuation of the pattern.

This mathematical disparity requires one to derive two distinct approaches to the orientation and the position of the dipole. To control the orientation, one simply needs to locally specify the desired electric-field angle. However, the position must be controlled by inducing motion through an applied force. Taking the negative gradient of the potential energy and assuming that the dipole and all sources are contained in the $x, y$ plane so that $\left.(\partial V / \partial z)\right|_{z=0}=0$, we obtain the force on the rod,

$$
\begin{aligned}
\mathbf{F}_{j}= & {\left.\left[2 \alpha_{\mathrm{rod}}\left(\frac{\partial V}{\partial x} \frac{\partial^{2} V}{\partial x^{2}}+\frac{\partial V}{\partial y} \frac{\partial^{2} V}{\partial x \partial y}\right) \mathbf{a}_{x}\right]\right|_{\mathbf{R}_{j}} } \\
& +\left.\left[2 \alpha_{\mathrm{rod}}\left(\frac{\partial V}{\partial x} \frac{\partial^{2} V}{\partial y \partial x}+\frac{\partial V}{\partial y} \frac{\partial^{2} V}{\partial y^{2}}\right) \mathbf{a}_{y}\right]\right|_{\mathbf{R}_{j}} .
\end{aligned}
$$

The electric field can be defined in polar coordinates at the location of a rod by a magnitude $E_{0}$ and an angle $\theta_{j}$. Making this transformation and separating the two components of Eq. (6) yields

$$
\begin{aligned}
& F_{j x}=-\left.2 \alpha_{\mathrm{rod}} E_{0}\left[\cos \left(\theta_{j}\right) \frac{\partial^{2} V}{\partial x^{2}}+\sin \left(\theta_{j}\right) \frac{\partial^{2} V}{\partial x \partial y}\right]\right|_{\mathbf{R}_{j}}, \\
& F_{j y}=-\left.2 \alpha_{\mathrm{rod}} E_{0}\left[\cos \left(\theta_{j}\right) \frac{\partial^{2} V}{\partial y \partial x}+\sin \left(\theta_{j}\right) \frac{\partial^{2} V}{\partial y^{2}}\right]\right|_{\mathbf{R}_{j}} .
\end{aligned}
$$

Additionally, the first derivatives of the voltage field are now defined in terms of the orientation of the dipole and the magnitude of the applied electric field needed to hold that orientation,

$$
\begin{aligned}
& \cos \left(\theta_{j}\right) E_{0}=-\left.\frac{\partial V}{\partial x}\right|_{\mathbf{R}_{j}}, \\
& \sin \left(\theta_{j}\right) E_{0}=-\left.\frac{\partial V}{\partial y}\right|_{\mathbf{R}_{j}} .
\end{aligned}
$$


For a dipole at $\mathbf{R}_{j}$, there are now four conditions through Eqs. (7) and (8) that the field must locally satisfy based on the desired orientation of the dipole, an applied field magnitude, and a force vector.

Having examined the force and orientation of a single rod in a local electric field, we return to the problem of controlling an ensemble of rods, each at a position $\mathbf{R}_{j}$, where $j$ goes from 1 to $N$. To satisfy each of the four constraints for each of the $N$ dipoles, one would generally require at least $4 N$ independent parameters that can be individually specified. This is accomplished by creating the electric field using $n$ electrodes where $n \geqslant 4 N$. Let us assume that each electrode $i$ can be approximated as a point charge $\left(q_{i}\right)$ and an induced dipole $\left(p_{x i}\right.$ and $\left.p_{y i}\right)$ due to the fields generated by all other electrodes. Although it is ultimately the voltage on each electrode that will be experimentally controlled, it is more tractable to use those sources as mathematically intermediate quantities. The contributions of the charge and dipole at $\mathbf{r}_{i}$ to the voltage field at $\mathbf{P}_{k}$ follow as

$$
\begin{aligned}
& q_{i} G_{q}\left[\mathbf{P}_{k}, \mathbf{r}_{i}\right]=q_{i} \frac{1}{4 \pi \varepsilon\left|\mathbf{P}_{k}-\mathbf{r}_{i}\right|}, \\
& p_{x i} G_{p_{x}}\left[\mathbf{P}_{k}, \mathbf{r}_{i}\right]=p_{x i} \frac{\left(\mathbf{P}_{k}-\mathbf{r}_{i}\right) \cdot \mathbf{a}_{x}}{4 \pi \varepsilon\left|\mathbf{P}_{k}-\mathbf{r}_{i}\right|^{3}},
\end{aligned}
$$

and

$$
p_{y i} G_{p_{y}}\left[\mathbf{P}_{k}, \mathbf{r}_{i}\right]=p_{y i} \frac{\left(\mathbf{P}_{k}-\mathbf{r}_{i}\right) \cdot \mathbf{a}_{y}}{4 \pi \varepsilon\left|\mathbf{P}_{k}-\mathbf{r}_{i}\right|^{3}}
$$

Note that $\mathbf{P}_{k}$ could be either the location of another electrode $\left(\mathbf{r}_{k}\right)$ or a $\operatorname{rod}\left(\mathbf{R}_{k}\right)$. The induced dipole on an electrode $i$ is proportional to the electric field at $\mathbf{r}_{i}$. Therefore, all the charges and dipoles are interrelated and the following relations must be satisfied in order to create a self-consistent system. For $i$ ranging from 1 to $n, \mathbf{p}_{i}=\left.\boldsymbol{\alpha}_{i} \cdot \mathbf{E}\right|_{\mathbf{r}_{i}}$ must be true. Consequently, by examining $x$ and $y$ components of the electric field, all the sources are constrained by

$$
\begin{aligned}
0= & \frac{p_{x i}}{\alpha_{x i}}+\sum_{\substack{j=1 \\
j \neq i}}^{n}\left(q_{j} \partial_{x} G_{q}\left[\mathbf{r}_{i}, \mathbf{r}_{j}\right]+p_{x j} \partial_{x} G_{p_{x}}\left[\mathbf{r}_{i}, \mathbf{r}_{j}\right]\right. \\
& \left.+p_{y j} \partial_{x} G_{p_{y}}\left[\mathbf{r}_{i}, \mathbf{r}_{j}\right]\right), \\
0= & \frac{p_{y i}}{\alpha_{y i}}+\sum_{\substack{j=1 \\
j \neq i}}^{n}\left(q_{j} \partial_{y} G_{q}\left[\mathbf{r}_{i}, \mathbf{r}_{j}\right]+p_{x j} \partial_{y} G_{p_{x}}\left[\mathbf{r}_{i}, \mathbf{r}_{j}\right]\right. \\
& \left.+p_{y j} \partial_{y} G_{p_{y}}\left[\mathbf{r}_{i}, \mathbf{r}_{j}\right]\right) .
\end{aligned}
$$

While the charge $q_{i}$ and two-dimensional (2D) dipole vector $\mathbf{p}_{i}$ on each electrode originally constituted a set of $3 n$ independent variables, Eq. (12) now provides $2 n$ equations relating the induced dipoles on a given electrode to the charge and dipoles existing on the other ones. We now require an additional set of $n$ equations.

Let us define the approximate voltage at the location $\mathbf{R}_{j}$ of a given particle as

$$
V\left[\mathbf{R}_{j}\right]=\sum_{i=1}^{n}\left(q_{i} G_{q}\left[\mathbf{R}_{j}, \mathbf{r}_{i}\right]+p_{x i} G_{p_{x}}\left[\mathbf{R}_{j}, \mathbf{r}_{i}\right]+p_{y i} G_{p_{y}}\left[\mathbf{R}_{j}, \mathbf{r}_{i}\right]\right)
$$

where it was assumed that $\mathbf{R}_{j}$ is located at some reasonable distance from all electrodes. For each rod $j$ ranging from 1 to $N$, employing the above approximation in Eqs. (7) and (8) yields

$$
\begin{aligned}
& \cos \left(\theta_{j}\right) E_{0}=-\partial_{x} V\left[\mathbf{R}_{j}\right], \\
& \sin \left(\theta_{j}\right) E_{0}=-\partial_{y} V\left[\mathbf{R}_{j}\right],
\end{aligned}
$$

and

$$
\begin{aligned}
& F_{j x}=-2 \alpha_{\text {rod }} E_{0}\left(\cos \left(\theta_{j}\right) \partial_{x, x} V\left[\mathbf{R}_{j}\right]+\sin \left(\theta_{j}\right) \partial_{x, y} V\left[\mathbf{R}_{j}\right]\right), \\
& F_{j y}=-2 \alpha_{\text {rod }} E_{0}\left(\cos \left(\theta_{j}\right) \partial_{y, x} V\left[\mathbf{R}_{j}\right]+\sin \left(\theta_{j}\right) \partial_{y, y} V\left[\mathbf{R}_{j}\right]\right) .
\end{aligned}
$$

Initially employing four times as many electrodes as there are rods (i.e., $n=4 N$ ), Eqs. (12), (14), and (15) result in a system of $3 n$ equations and $3 n$ unknowns that can be numerically solved for the charge and dipole on each of the $n$ electrodes.

While $n=4 N$ electrodes will generally satisfy those conditions, some pathological situations do exist that would actually require using $n>4 N$ electrodes. Consider a matrix, $\mathbf{M}$, formed from the coefficients of the right-hand sides of Eqs. (12), (14), and (15). When the determinant of $\mathbf{M}$ is zero, it will be impossible to find a set of charges and dipoles satisfying an arbitrary orientation and force vector. This matrix is a function of the position and the orientation of the rod and yields a whole region in which no solution exists for some combination of position and orientation when solely using $4 N$ electrodes to specify the orientation and force of a single $(N=1)$ particle (Fig. 2). On the other hand, if there are $n$ $=4 N+x$ electrodes, one can generate $x$ additional constraints. These additional constraints maintain a linear relationship between the sources at each electrode and the field conditions to be met at each dipole. In fact, they may be fairly arbitrary as long as they do not directly conflict with the conditions stated previously. With this in mind, we thus simply add an additional electrode so that $n=4 N+1$, and introduce the additional constraint of

$$
0=\sum_{i=1}^{n} q_{i}
$$

By doing so, we find no combination of position and orientation around the electrodes in which $\|\mathbf{M}\|=0$ (Fig. 3).

These charges and dipoles are then used to determine the set of voltages to be applied to the electrodes. Providing that the capacitance of the electrode $i$ is $C_{i}$, the voltage on each electrode $V_{i}$ is 


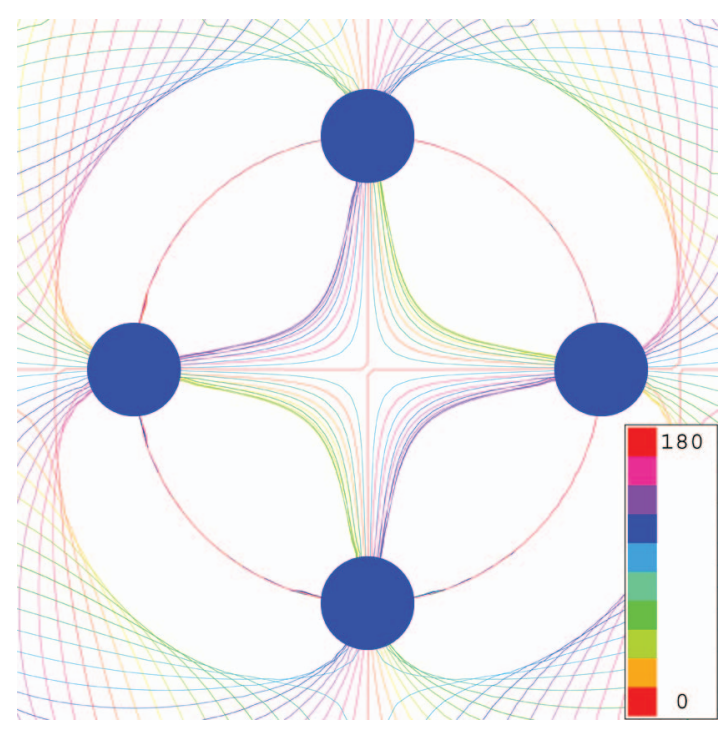

FIG. 2. (Color) To find the voltage on each electrode which creates the desired angle and movement of a nanorod, one must solve a system of constraints $\boldsymbol{M}$ which is dependent on only the angle and position of the nanorod. While generally four electrodes are satisfactory to control a single rod, for each angle $\theta$ there exists a set of lines in the $x-y$ plane for which it will be impossible to apply an arbitrary force (i.e., $\|\mathbf{M}\|=0$ ). In the plot above, these lines are overlaid on each other as the angle of the rod is changed in discreet steps. The electrode diameter is $20 \mu \mathrm{m}$.

$$
\begin{aligned}
V_{i}= & q_{i} / C_{i}+\sum_{\substack{j=1 \\
j \neq i}}^{n}\left(q_{j} G_{q}\left[\mathbf{r}_{i}, \mathbf{r}_{j}\right]+p_{x j} G_{p_{x}}\left[\mathbf{r}_{i}, \mathbf{r}_{j}\right]\right. \\
& \left.+p_{y j} G_{p_{y}}\left[\mathbf{r}_{i}, \mathbf{r}_{j}\right]\right)
\end{aligned}
$$

where $i$ ranges go from 1 to $n$.

\section{ESTIMATION OF SUITABLE SIMULATION PARAMETERS}

We will now demonstrate that this approach to the manipulation of individual particles can be practically implemented using a realistic range of parameters. In addition to the geometry of the electrodes, experimental parameters such as the dipole's polarizability $\left(\alpha_{\text {rod }}\right)$, the applied force $\left(F_{x}\right.$ and $\left.F_{y}\right)$, and the applied electric field $\left(E_{0}\right)$ must be specified. We consider the manipulation of a gold nanorod as a suitable test case for such purposes.

Consider a gold nanorod with a length $l=1.4 \mu \mathrm{m}$, a radius $r=35 \mathrm{~nm}$, and a conductivity $\sigma_{\text {rod }}=\infty$, suspended in deionized water at room temperature with a dynamic viscosity $\mu_{\text {medium }}=1.0 \mathrm{mPa} \mathrm{s}$, a dielectric constant $\varepsilon_{\text {medium }}=80 \varepsilon_{0}$, and a conductivity $\sigma_{\text {medium }}=5.5 \times 10^{-6}(\mathrm{~m} \Omega)^{-1}$.

Providing that the signal applied to all electrodes is square waves at a frequency $f_{\text {carrier }} \gg \sigma_{\text {medium }} / 2 \pi \varepsilon_{\text {medium, }}$, the conductivity of the medium can be ignored and the water treated as a pure dielectric without adding the complication of time-average fields to the derivation. While the employment of ac sources may initially seem as a departure from the theory developed in previous section, a close examination of Eqs. (12), (14), (15), and (17) would show that flipping the polarity of all voltages $V_{i}$ will result in the same dipole orientation and net applied force. Intuitively speaking, an in-
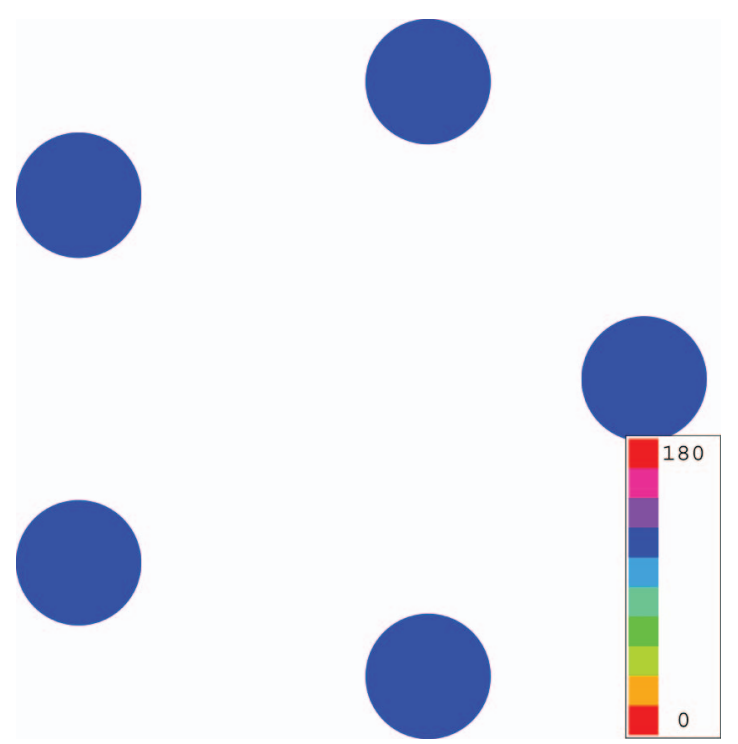

FIG. 3. (Color) In the four electrode structure there exists a region in which for some angle $\theta$ it will be impossible to control the rod. To illustrate that no such region exists in the five electrode structure, the angle of the rod is varied in discreet steps while looking for the lines in which $\|\mathbf{M}\|=0$. In the five electrode structure these lines do not exists. The electrode diameter is $20 \mu \mathrm{m}$.

duced dipole is oriented and attracted by a point charge in the same way regardless of its polarity. Finally, the problem can be treated under a "quasistatic" regime as long as the wavelength $\lambda=c / f_{\text {carrier }}$ remains much larger than any other experimental dimension. Employing square waves as sources therefore solely serves to eliminate the medium conductivity from the development. For de-ionized water $f_{\text {carrier }}$ should be greater than $1.2 \mathrm{kHz}$. Similar reasonable values can be found for organic solvents. However, cell culture media with conductivities on the order of $2(\mathrm{~m} \Omega)^{-1}$ will require $f_{\text {carrier }}$ to be greater than $450 \mathrm{MHz}$. While such range remains readily available with standard rf generators, such necessity would incur higher implementation costs due to the higher prices of such equipment.

Using a derivation found elsewhere ${ }^{21}$ and further approximating the rod as a prolate ellipsoid perfect conductor in a dielectric, the polarizability along the main axis yields

$$
\alpha_{\mathrm{rod}}=\frac{4 l r^{2} e^{3} \pi \varepsilon_{\text {medium }}}{3\left(e^{2}-1\right)(2 e-\ln [1+e / 1-e])},
$$

where $e=\sqrt{1-r^{2} /(l / 2)^{2}}$. For the particle size and conditions presented, this yields a polarizability of $\alpha_{\text {rod }}=3$ $\times 10^{-27} \mathrm{C} \mathrm{m}^{2} / \mathrm{V}$.

One would generally wish to apply a force generating a predetermined translational velocity to the rod. The viscous drag coefficients relate the two and thus allows us to estimate a reasonable range of required applied force. ${ }^{22}$ The rod has drag coefficients such that for a fluid flow velocity $V_{\|}$along its axis,

$$
F_{D \|}=V_{\|} C_{\|}=V_{\|} \frac{2 \pi \mu_{\text {medium }} l}{\ln \left[\frac{l}{r}\right]-0.72},
$$

and for a fluid flow velocity $V_{\perp}$ normal to its axis, 


$$
F_{D \perp}=V_{\perp} C_{\perp}=V_{\perp} \frac{2 \pi \mu_{\text {medium }} l}{\ln \left[\frac{l}{r}\right]+0.5} .
$$

For the particle size and conditions presented, this yields $C_{\|}=5.9 \times 10^{-9} \mathrm{~N} \mathrm{~s} / \mathrm{m}$ and $C_{\perp}=5.9 \times 10^{-9} \mathrm{~N} \mathrm{~s} / \mathrm{m}$. It is worth noting that the drag coefficients are relatively close in spite of an aspect ratio of 20. Defining one rod length per second as a reasonable desired velocity, and using the parallel drag coefficient in Eq. (19) yields a required force of $F_{\text {norm }}$ $=4.1 \mathrm{fN}$. The steady-state velocity is reached very quickly so that one can consider the induced velocity as directly proportional to the applied force through the drag coefficients. It is therefore reasonable to take $F_{x}$ and $F_{y}$ to be some large fraction of $F_{\text {norm }}$ to maintain a velocity of a little less than one rod length per second.

The magnitude of the electric field has several constraints. The field must be strong enough to rotate the rod against viscous drag, hold the rod's orientation against Brownian motion, and hold the rod in the $z=0$ plane.

Ignoring Brownian motion, the slightest electric field will eventually orient the nanorod along the desired orientation. However, if the desired angle of the rod is changing and the electric field is too weak, viscous drag will prevent rod from orienting with the field. Let us therefore assume that we wish to change the orientation at a minimal rotational velocity of $f=1 \mathrm{~s}^{-1}$ and can tolerate a phase lag between the rod and the electric field of $\phi=\pi / 8$. The torque due to the electric field is dependent on the magnitude of the electric field and the phase difference between the rod and the field. Noting that the rod is an induced dipole so only the component of the field along the major axis contributes to $p_{\text {rod }}$, the electric torque is given as

$$
\left|T_{E}\right|=p\left|E_{0}\right| \sin [\phi]=\alpha_{\text {rod }}\left|E_{0}\right|^{2} \cos [\phi] \sin [\phi] .
$$

The torque due to drag on a rod rotating at a frequency $f$ can be estimated from Eq. (20) as

$$
T_{D}=\int_{-l / 2}^{l / 2} \frac{4 \pi \mu_{\text {medium }}(2 \pi f R)}{\ln [l / r]+0.5} R d R=\frac{2 l^{3} f \pi^{2} \mu_{\text {medium }}}{3\left(\ln \left[\frac{l}{r}+0.5\right]\right)}
$$

Assuming steady-state conditions, setting Eqs. (21) and (22) equal to each other and solving for the electric field yield a lower bound on the electric field of

$$
E_{0}>E_{\mathrm{rot}}=\sqrt{\frac{2 l^{3} f \pi^{2} \mu_{\text {medium }}}{3 \alpha_{\mathrm{rod}} \cos (\phi) \sin (\phi)[\ln (l / r)+0.5]}} .
$$

For the conditions proposed, the value of the minimum electric field to needed to rotate the rod against viscous forces is $E_{\text {rot }}=6.0 \mathrm{kV} / \mathrm{m}$.

Since the polarizability scales with the cube of the particle size, this value is unaffected by the particle size and depends only on the particle shape. However, for nanoparticles Brownian motion can become a significant factor. Within the fluid, energy is indeed exchanged randomly between the molecules and the particles with amounts on the order of $\Delta E=k_{B} T$, where $k_{B}$ is Boltzmann's constant, and $T$ is the temperature in Kelvin. At room temperature, this value is $\Delta E=4.1 \times 10^{-21} \mathrm{~J}$. The effects of Brownian motion are therefore estimated by comparing this thermal fluctuation energy to the energy involved in a related displacement from electrostatic equilibrium. ${ }^{9}$ In other words, the potential-energy difference between the desired angle and a maximum tolerable displacement angle must be smaller than $\Delta E$ in order to maintain the particle's orientation against Brownian motion.

The potential energy of the nanorod oriented at an angle $\gamma$ to the electric field is $U(\gamma)=-\alpha_{\text {rod }} E_{0}{ }^{2} \cos (\gamma)^{2}$. Note that if $\gamma=0$, then the rod is in equilibrium, aligned with $\theta$, and in agreement with Eq. (1). If an angle $\gamma_{\max }=\pi / 4$ is defined as the maximum tolerable displacement from $\theta$, the thermal energy required for such a displacement is $\Delta E=U\left(\gamma_{\max }\right)$ $-U(0)$. This yields a lower bound on the electric field of

$$
E_{0}>E_{\text {orient }}=\frac{1}{\sin \left(\gamma_{\max }\right)} \sqrt{\frac{k_{B} T}{\alpha_{\text {rod }}}} .
$$

Using the given temperature, polarizability, and maximum allowable displacement, the minimum electric field to hold the rod's orientation is $E_{\text {orient }}=4.7 \mathrm{kV} / \mathrm{m}$.

While lateral ( $x$ and $y$ ) Brownian motion can be managed by applying small forces counter to the drift, vertical $(z)$ Brownian motion cannot be directly compensated. However, the electric field will decay with distance from the electrodes and therefore the plane $z=0$ is a weak positive dielectrophoretic trap with respect to the $z$ axis. Quantifying this trap is difficult because of the complex nature of the electric field generated by the electrodes. However, if each electrode is represented by only a point charge and assumed to lie on a circle of radius $a$, so that the voltage field is

$$
V[\mathbf{R}]=\sum_{i=1}^{n} \frac{q_{i}}{4 \pi \varepsilon\left|\mathbf{R}-a\left\{\cos \left(\kappa_{i}\right), \sin \left(\kappa_{i}\right), 0\right\}\right|},
$$

and we further assume that the particle is at the center of the array, than it is easily shown that the potential energy of the rod is

$$
U[\{0,0, z\}]=-\left\{\frac{1}{\left[1+(z / a)^{2}\right]^{4}}\right\} \alpha_{\mathrm{rod}} E_{0}^{2} .
$$

Again, comparing the thermal energy to the difference in potential energy between the equilibrium position and a maximum tolerable displacement $z_{\max }=2 l$ yields $\Delta E$ $=U\left(z_{\max }\right)-U(0)$. Solving for the electric field presents the condition

$$
E_{0}>E_{\mathrm{z} \text { axis }}=\left\{1-\frac{1}{\left[1+\left(z_{\max } / a\right)^{2}\right]^{4}}\right\}^{-1 / 2} \sqrt{\frac{k_{B} T}{\alpha_{\text {rod }}}} .
$$

For the electrodes spaced on a circle with a radius $a$ $=25 \mu \mathrm{m}$ and the conditions proposed, the minimum electric field to keep the rods near to $x, y$ plane is $E_{z \text { axis }}=15 \mathrm{kV} / \mathrm{m}$. Since this is the most stringent constraint, the electric field required at the particle location is also $E_{0}=15 \mathrm{kV} / \mathrm{m}$.

If the thermal constraints $\left(E_{\text {orient }}\right.$ or $\left.E_{z \text { axis }}\right)$ are more stringent than the rotational constraint $\left(E_{\text {rod }}\right)$, and the particle size is linearly scaled down, the electric field must grow with a power of $3 / 2$ to compensate. On the other hand, if the 

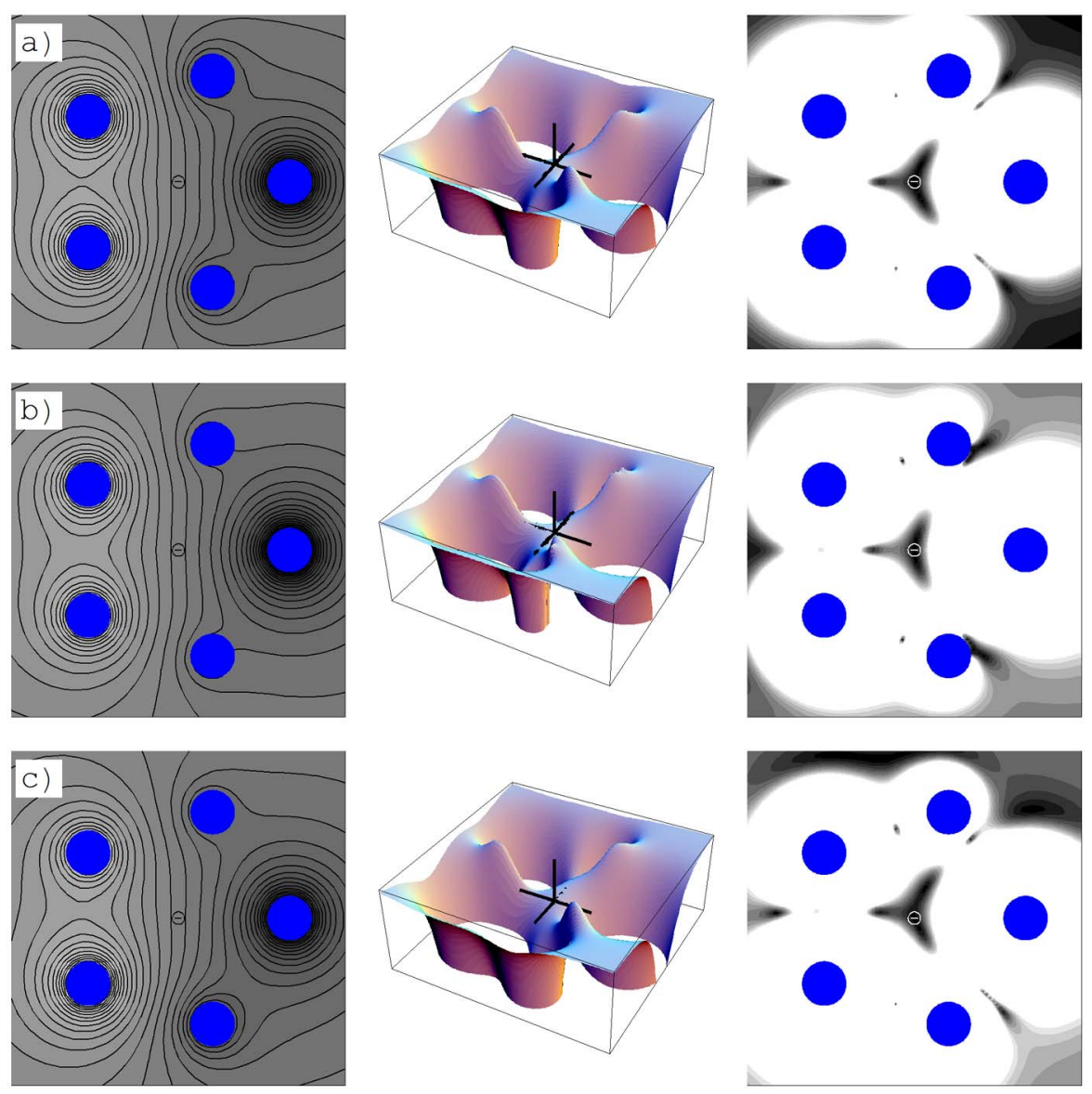

FIG. 4. (Color online) Voltage field, potential energy, and relative error in force in the plane of five electrodes. The rod is at the center angled with the $x$ axis for each set. It is indicated by the $1.4 \mu \mathrm{m}$ line emphasized by the circle in the first and third columns and by the cross in the second column. The force on the rod is negligible, oriented toward $+x$, and oriented toward $-y$ for (a), (b), and (c), respectively. The electrode diameter is $20 \mu \mathrm{m}$. The voltages range from -3 (black) to $+3 \mathrm{~V}$ (white) in the first column and the relative error ranges from 0 (black) to 1 (white). electrode array is linearly scaled down with the particle, the voltage on the electrodes must only grow with a power of $1 / 2$ in order to compensate.

For simulation purposes, we will therefore consider $15 \mathrm{kV} / \mathrm{m}$ as the required applied electric field, and some fraction of $4.1 \mathrm{fN}$ as the applied force. Additionally, we will use an array of five spherical electrodes located in the $x, y$ plane, centered on a circle with a diameter of $50 \mu \mathrm{m}$, each with a diameter of $10 \mu \mathrm{m}$. However, these ideas easily extrapolate to other planar structures that would be readily fabricated using standard lithography techniques.

\section{DISCUSSION OF SIMULATION RESULTS}

In Fig. 4, parts (a), (b), and (c) show a dipole held at the origin with an orientation of $\theta=0$ by a field of $15 \mathrm{kV} / \mathrm{m}$ with force vectors of $\{0,0\},\{2,0\},\{0,-2\} \mathrm{fN}$, respectively. The first image in each part of Fig. 4 shows the voltage field in the plane. The false color scale ranged from -3 to $3 \mathrm{~V}$, which is easily realized on standard wave form generator. It is therefore clear that no unreasonable voltages are required. The dipole will lie with the electric field, perpendicular to the lines of constant voltage. In each part of Fig. 4, it is also apparent that this orients the rod at $\theta=0$, as predicted.

The second image in each part of Fig. 4 shows the dipole potential energy $-\alpha_{\text {rod }} \mathbf{E} \cdot \mathbf{E}$. The dipole lies at the center. The dipole will move in the direction in decreasing potential energy. In Fig. 4(a), the center represents an unstable balance point with no net force. In Fig. 4(b), the potential energy is sloped in the $+x$ direction, and in Fig. 4(c) the potential energy is sloped in the $-y$ direction as predicted.

The third column shows the magnitude of difference in force between what was desired and what exists at a particular location normalized by the $F_{\text {norm, }}$ i.e., $\mid \mathbf{F}[\{x, y\}]$ $-\mathbf{F} \mid / F_{\text {norm. }}$. As discussed earlier, even in Fig. 4(a) where the force is zero, the center is a point of instability. This is readily apparent in the plots of the potential energy. Regions in white represent errors greater than one and correspond to the rod moving at a velocity greater than one rod length per second different than what was desired. In each set, there is a region several rod lengths across in which the error is substantially less than one, indicating that the rod's movement away from that point will be slow enough to allow active or user feedback to be used to control motion. This allows the rod to be balanced at that point if no motion is desired or moved in a controlled fashion if a force is applied.

\section{SUMMARY}

We derived and examined a method to arbitrarily move and orient an ensemble of metallic rod-shaped particles using static electric fields. Using the control of a single nanorod as an example, we find suitable parameters, and simulate the algorithm. The results show that the desired orientation and force is produced for a small distance around the rod. While the results also indicate that the system is unstable with regard to position, it is not so unstable as to prevent compen- 
sation through a simple feedback mechanism. This could provide a simple "touchless" approach to the planar manipulation of nanostructures.

\section{ACKNOWLEDGMENTS}

One of the authors (B.E.) would like to thank NSF IGERT Grant No. DGE-0221664 for their continued support and the authors would like to acknowledge NSF NIRT Grant No. 0303981.

${ }^{1}$ P. A. Smith, C. D. Nordquist, T. N. Jackson, T. S. Mayer, B. R. Martin, J. Mbindyo, and T. E. Mallouk, Appl. Phys. Lett. 77, 1399 (2000).

${ }^{2}$ A. Castellanos, A. Ramos, A. Gonzalez, N. G. Green, and H. Morgan, J. Phys. D 36, 2584 (2003).

${ }^{3}$ T. Muller, A. Pfennig, P. Klein, G. Gradl, M. Jager, and T. Schnelle, IEEE Eng. Med. Biol. Mag. 22, 51 (2003).

${ }^{4}$ K. C. Neuman and S. M. Block, Rev. Sci. Instrum. 75, 2787 (2004).

${ }^{5}$ W. H. Wright, G. J. Sonek, Y. Tadir, and M. W. Berns, IEEE J. Quantum Electron. 26, 2148 (1990).

${ }^{6}$ T. R. Ramachandra, C. Baur, A. Bugacov, A. Madhukar, B. E. Koel, A. Requicha, C. Gazen, Nanotechnology 9, (1998).

${ }^{7}$ C. Gosse and V. Croquette, Biophys. J. 82, 3314 (2002).
${ }^{8}$ M. P. Hughes, Nanotechnology 11, 124 (2000).

${ }^{9}$ N. G. Green, A. Ramos, and H. Morgan, J. Phys. D 33, 632 (2000).

${ }^{10}$ A. Ramos, H. Morgan, N. G. Green, and A. Castellanos, J. Phys. D 31, 2338 (1998).

${ }^{11}$ H. A. Pohl, Dielectrophoresis: The Behavior of Neutral Matter in Nonuniform Electric Fields (Cambridge University Press, Cambridge, 1978).

${ }^{12}$ J. Voldman, R. A. Braff, M. Toner, M. L. Gray, and M. A. Schmidt, Biophys. J. 80, 531 (2001).

${ }^{13}$ T. Schnelle, T. Muller, and G. Fuhr, J. Electrost. 50, 17 (2000).

${ }^{14}$ T. Muller, G. Gradl, S. Howitz, S. Shirley, T. Schnelle, and G. Fuhr, Biosens. Bioelectron. 14, 247 (1999).

${ }^{15}$ C. Reichle, T. Muller, T. Schnelle, and G. Fuhr, J. Phys. D 32, 2128 (1999).

${ }^{16}$ T. Schnelle, T. Muller, R. Hagedorn, A. Voigt, and G. Fuhr, Biochim. Biophys. Acta 1428, 99 (1999).

${ }^{17}$ J. Suehiro and R. Pethig, J. Phys. D 31, 3298 (1998).

${ }^{18}$ T. B. Jones and J. P. Kraybill, J. Appl. Phys. 60, 1247 (1986).

${ }^{19}$ E. G. Cen, C. Dalton, Y. L. Li, S. Adamia, L. M. Pilarski, and K. Kaler, J. Microbiol. Methods 58, 387 (2004).

${ }^{20}$ D. L. Fan, F. Q. Zhu, R. C. Cammarata, and C. L. Chien, Appl. Phys. Lett. 85, 4175 (2004).

${ }^{21}$ A. H. Sihvola, Electromagnetic Mixing Formulas and Applications (Institution of Electrical Engineers, London, 1999).

${ }^{22}$ J. M. Saleh, Fluid Flow Handbook (McGraw-Hill, New York, 2002). 